- Regular Articles-

\title{
Improvement in Intestinal Coenzyme Q10 Absorption by Food Intake
}

\author{
Akiko Ochiai, Shirou Itagaki, Toshimitsu Kurokawa, \\ Masaki KoBAYASHI, Takeshi HIRANO, and Ken ISEKI* \\ Laboratory of Clinical Pharmaceutics \& Therapeutics, Division of Pharmaceutics, Faculty \\ of Pharmaceutical Sciences, Hokkaido University, Kita-12-jo, \\ Nishi-6-chome, Kita-ku, Sapporo 060-0812, Japan
}

(Received February 3, 2007; Accepted May 30, 2007; Published online June 1, 2007)

\begin{abstract}
Coenzyme Q10 (CoQ10) is widely consumed as a food supplement because of its recognition as an important nutrient in supporting human health. Absorption of compounds from the gastrointestinal tract is one of the important determinants of oral bioavailability. However, the absorption of dietary CoQ10 is slow and limited due to its hydrophobicity and large molecular weight. The absorption of orally applied compounds can be enhanced by interactions with food or food components. Thus, we investigated the effect of food intake on the absorption of CoQ10 after oral supplementation. In this study, we demonstrated that food intake enhanced the intestinal absorption of CoQ10. In order to improve intestinal absorption of CoQ10 after oral supplementation, we developed an emulsion formulation. Intestinal absorption of CoQ10 after administration of the emulsion formulation was also enhanced by food intake. Moreover, the peak concentration and the extent of absorption after administration of the emulsion formulation were greater than those after administration of a suspension formulation. It is possible that administration of CoQ10 in an emulsion formulation enhances the pharmacological effects of CoQ10.
\end{abstract}

Key words_ coenzyme; bioavailability; food; emulsion

\section{INTRODUCTION}

Coenzyme Q10 (CoQ10) functions in its reduced form as an antioxidant, protecting biological membranes and serum LDL from lipid peroxidation. ${ }^{1-3)}$ CoQ10 is a ubiquitous compound vital to a number of activities related to energy metabolism. Humans, by nature, have the ability to produce CoQ10. However, this ability starts to decline at the age of 20 years and the amount of CoQ10 in the body decreases rapidly after the age of 40 years. ${ }^{4)}$ The importance of CoQ10 for living organisms has been illuminated by reports of genetic disorders in which CoQ10 synthesis is impaired. ${ }^{5)}$

CoQ10 is widely consumed as a food supplement because of its recognition as an important nutrient in supporting human health. The rationale for the use of CoQ10 as a therapeutic agent in cardiovascular and degenerative neurologic and neuromuscular diseases is based on its fundamental role in mitochondrial function and cellular bioenergetics. There are data supporting the therapeutic value of $\mathrm{CoQ} 10$ as an adjust to standard medical therapy in cardiovascular diseases. ${ }^{6-8)}$ There are also data indicating beneficial

\footnotetext{
*e-mail: ken-i@pharm.hokudai.ac.jp
}

effects of CoQ10 in patients with diabetes and cancer. ${ }^{9,10)}$

Absorption of compounds from the gastrointestinal tract is one of the important determinants of oral bioavailability. However, the absorption of dietary CoQ10 is slow and limited due to its hydrophobicity and large molecular weight. ${ }^{11)}$ The absorption of orally applied compounds can be enhanced by interactions with food or food components. Thus, we focused on the effect of food intake on the absorption of CoQ10 after oral supplementation. However, there are various effects of food intake on drug absorption. Food intake has been found to enhance absorption in some cases but to weaken absorption in other cases. The aim of this study was to determine the influence of food intake on the pharmacokinetics of CoQ10 after oral administration. In order to improve intestinal absorption of CoQ10 after oral supplementation, we developed an emulsion formulation. We investigated the effect of food intake on the pharmacokinetics of CoQ10 after oral administration of the emulsion formulation.

\section{MATERIALS AND METHODS}

Chemicals CoQ10 powder and emulsion formulation of CoQ10 were kindly supplied by Kougen 
Co., Ltd. (Shizuoka, Japan: manufactured by Zhejiang Medicine Co., Ltd. Xinchang Pharmaceutical Factory). All other reagents were of the highest grade available and used without further purification.

Animals Male Wistar rats, aged 7 to 9 weeks (200-250 g in weight), were obtained from Jla (Tokyo, Japan). The housing conditions were the same as those described previously. ${ }^{12)}$ The rats were housed at least 1 week at $23 \pm 3{ }^{\circ} \mathrm{C}$ and $50 \pm 10 \%$ relative humidity and were maintained on a $12 \mathrm{~h}$ light/ dark cycle. During the acclimatization the rats were allowed free access to food and water. The experimental protocols were reviewed and approved by the Hokkaido University Animal Care Committee in accordance with the "Guide for the Care and Use of Laboratory Animals",.

In vivo Administration Study To determine the effect of food-intake on the absorption of CoQ10, the rats were divided into two groups: a control group in which rats were fasted for $14 \mathrm{~h}$ prior to the experiments and a food-intake group in which rats were allowed free access to food. Rats were anaesthetized by an intraperitoneal (i.p.) injection of $50 \mathrm{mg} / \mathrm{kg}$ sodium pentobarbital. The rats were fixed after the operation. CoQ10 was administered in a suspension or emulsion ( $25 \mathrm{mg} / \mathrm{kg}$ body weight). Sequential blood samples were obtained from the femoral vein. Plasma was obtained by centrifugation ( $850 \mathrm{x} \mathrm{g}$ for $10 \mathrm{~min}$ ).

In situ Absorption Study Rats were fasted for $14 \mathrm{~h}$ prior to the experiments and a food-intake group in which rats were allowed free access to food. Rats were anaesthetized by an i.p. injection of $50 \mathrm{mg} / \mathrm{kg}$ sodium pentobarbital. The rats were fixed after the operation. A small midline incision was made in the abdomen. A 10-cm-long loop of the jejunum was identified and ligated at both ends. Five hundred $\mu \mathrm{l}$ of CoQ10 $(2.5 \mathrm{mg} / \mathrm{ml}$ of CoQ10) was administered directly into the loops. Intestinal contents were taken from the loops at $30 \mathrm{~min}$ after injection.

Analytical Procedures Coenzyme Q10 was determined by HPLC using absolute calibration curve method. HPLC method and sample processing were modified as described by $\mathrm{Lu}$ et al. ${ }^{13}$ ) with minor modification. Two hundred $\mu \mathrm{l}$ of CoQ10 solutions in absolute methanol was added to $100 \mu$ l of plasma to prepare the calibration curves. One hundred $\mu \mathrm{l}$ of specimens was diluted three fold with methanol. After vortexing, the sample was extracted with $1 \mathrm{ml}$ of n-hexane. After shaking the mixture vigorously, the sample was centrifuged at $2000 \mathrm{xg}$ for $5 \mathrm{~min}$ at $4^{\circ} \mathrm{C}$. Nine hundred $\mu \mathrm{l}$ of the organic layer was evaporated to dryness under a gas stream. The residue was redissolved in $100 \mu \mathrm{l}$ of mobile phase for HPLC injection. The concentration of CoQ10 was determined using an HPLC system equipped with a JASCO 880-PU pump and a 870-UV UV-vis detector. The column was a GL Science ODS-2 (5 $\mu \mathrm{m}$ in particle size, $4.6 \mathrm{~mm}$ in inside diameter x $250 \mathrm{~mm}$ ). A mobile phase containing 2-propanol/methanol/THF (55/39/6) was used. The column temperature and flow rate were $40^{\circ} \mathrm{C}$ and 1.0 $\mathrm{ml} / \mathrm{min}$, respectively. The wavelength for detection was $275 \mathrm{~nm}$. Forty $\mu \mathrm{l}$ of sample was injected into the HPLC system. We used CoQ10 powder for a standard solution. Calibration curve was constructed in the concentration range of $0-2.4 \mathrm{mg} / 1$. The absolute recoveries of CoQ10 were estimated by comparison of the area increments after extraction from plasma to that obtained after direct injection of a solution. CoQ10 extraction yielded significant recoveries (65 $\%$ ) and showed the best reproducibility.

Data Analysis A two-compartment model was fitted to the plasma data using Origin 6.1J. The parameters in this model are $D$ (dose of administration), $k_{21}$ (rate constant for transfer from the peripheral to central compartment, and $V$ (volume of distribution). The area under the plasma concentration-curve (AUC) was estimated by the trapezoidal rule. Student's $t$-test was used for statistical analysis, and a value of $p<0.05$ was considered significant.

\section{RESULTS AND DISCUSSION}

CoQ10 is a ubiquitous compound vital to a number of activities related to energy metabolism. Since dysfunctional energy metabolism has been shown to be a factor contributing to a number of conditions, dietary supplementation of CoQ10 has been used in the treatment of cardiac, neurologic, oncologic and immunologic disorders. ${ }^{14)}$ However, CoQ10 is taken up from the intestine at a low rate. ${ }^{11)}$ Since the absorption of orally applied compounds can be enhanced by the presence of food components, we focused on the effect of food intake on absorption of CoQ10.

In the first part of this study, we investigated the effect of food intake on plasma concentration of CoQ10 after single oral administration of CoQ10 in a suspension. Figure 1 shows the plasma concentration of CoQ10 after oral administration. Pharmacokinetic parameters are listed in Table 1 . The time to reach 


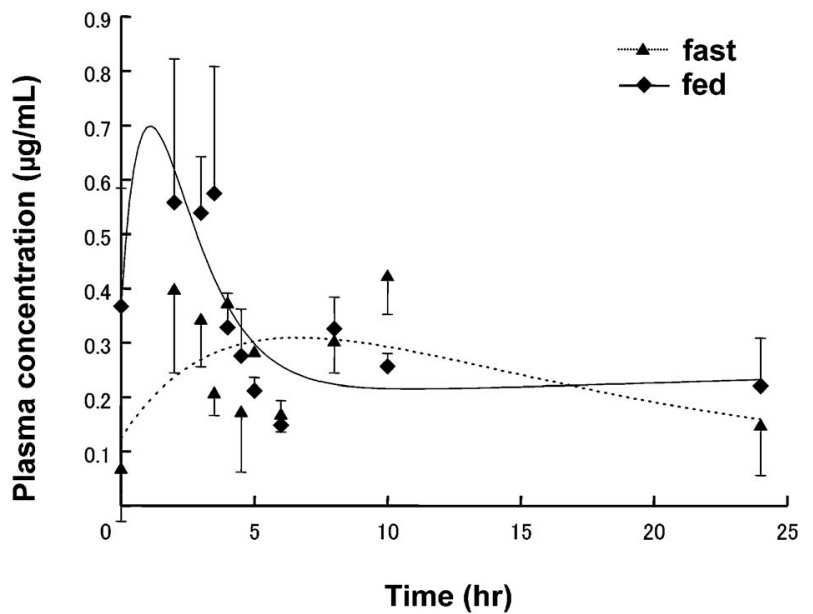

Fig. 1. Time Profile of Plasma Concentration of CoQ10 after Oral Administration ( $25 \mathrm{mg} / \mathrm{kg}$ Body Weight) of a Suspension

Each point represents the mean with S.E. of 3-6 measurements.

Table 1. Effect of Food Intake on Kinetic Parameters of CoQ10 after Oral Administration of a Suspension

\begin{tabular}{cccc}
\hline \hline & $C_{\max }(\mu \mathrm{g} / \mathrm{ml})$ & $T_{\max }(\mathrm{h})$ & $\operatorname{AUC}(\mu \mathrm{g} \mathrm{h} / \mathrm{ml})$ \\
\hline Fast & 0.31 & 3.64 & 3.99 \\
Fed & 0.70 & 1.13 & 6.86 \\
\hline
\end{tabular}

maximum $\left(T_{\max }\right)$ in the food-intake group was threetimes shorter than that in the control group. This finding suggests that intestinal absorption of CoQ10 is 3-fold faster with food intake. Moreover, the peak concentration $\left(C_{\max }\right)$ and AUC of the food-intake group were almost 2-fold greater than those of the control group. These results suggest that food intake enhanced the intestinal absorption of CoQ10.

Although the mechanism of uptake of CoQ10 has not been studied, solubility of compounds is one of the most critical issues. The use of a lipid-based formulation seems promising as a strategy to overcome the problem of poor solubility. ${ }^{15,16)}$ We therefore tried to improve the intestinal absorption of CoQ10 by using an emulsion formulation. In the second part of this study, we investigated the intestinal absorption of test formulations of CoQ10 in an in situ loop study. The intestinal absorption of CoQ10 is shown in Fig. 2. The residual amount of CoQ10 after administration in suspension was almost the same as the amount of CoQ10 administrated. This finding indicated that the absorption of CoQ10 after administration in a suspension is poor. The residual amount of CoQ10 after administration in an emulsion formula-

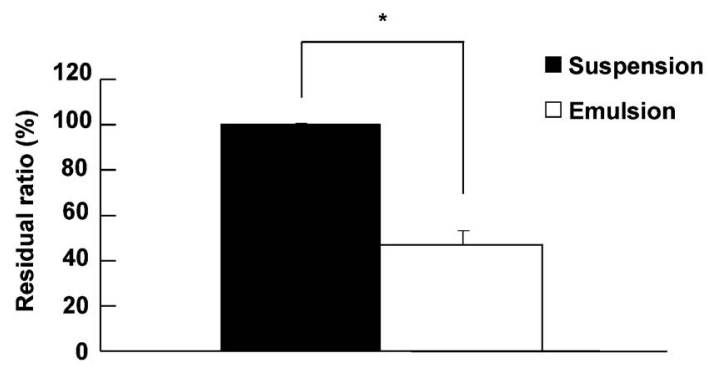

Fig. 2. Residual Ratio of CoQ10 in the Intestinal Loop

CoQ10 $(1.25 \mathrm{mg})$ was administered into the loop. Intestinal contents were taken from the loops at $30 \mathrm{~min}$ after injection. Each column represents the mean with S.E. of 3 measurements. ${ }^{*} p<0.05$, significantly different.

tion was significantly smaller than that after administration in a suspension. This result indicated that the absorption of CoQ10 was improved by using the emulsion formulation.

The above-described findings indicated the possibility that absorption of CoQ10 can be improved by using both an emulsion formulation and food intake. In the last part of this study, we therefore investigated the effect of food intake on the intestinal absorption of an emulsion formulation of CoQ10. Figure 3 shows the plasma concentration of CoQ10 after oral administration of the emulsion formulation. Pharmacokinetic parameters are listed in Table 2. $T_{\max }$ of the food-intake group was two-times shorter than that of the control group. This finding suggests that intestinal absorption of CoQ10 is 2-fold faster with food intake. Moreover, $C_{\max }$ and AUC of the foodintake group were 5-fold and 2 -fold greater than those of the control group, respectively. These results suggest that intestinal absorption of CoQ10 after administration of the emulsion formulation was also enhanced by food intake. $T_{\max }, C_{\max }$ and AUC after administration of the emulsion formulation in the foodintake group were three-times smaller, eight-times larger and five-times larger, respectively, than those after administration of the suspension in the control group. These findings suggest that the development of appropriate dosing regimens using emulsion formulation of CoQ10 with food supplementation may offer improved pharmacological effects. In addition to food components, bile acids are also known to enhance the intestinal absorption of poorly water-soluble drugs. Food intake stimulates biliary excretion of bile acids. This absorption process is carried out by micelles. The formation of micelles and incorporation of poorly water-soluble drugs into micelles are thought to be important for absorption. Since bile 


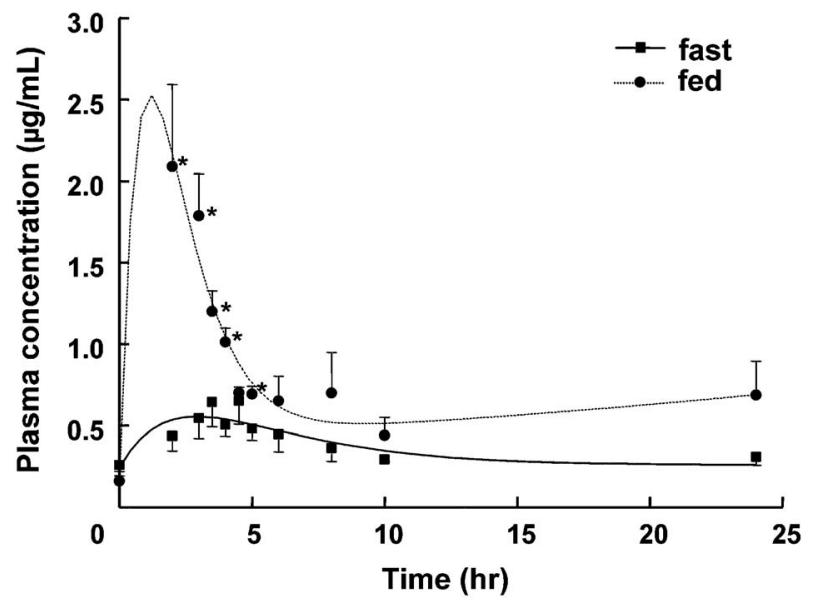

Fig. 3. Time Profile of Plasma Concentration of CoQ10 after Oral Administration ( $25 \mathrm{mg} / \mathrm{kg}$ Body Weight) of an Emulsion Formulation

Each point represents the mean with S.E. of 3 measurements. ${ }^{*} p<0.05$, significantly different.

Table 2. Effect of Food Intake on Kinetic Parameters of CoQ10 after Oral Administration of an Emulsion Formulation

\begin{tabular}{cccc}
\hline \hline & $C_{\max }(\mu \mathrm{g} / \mathrm{ml})$ & $T_{\max }(\mathrm{h})$ & $\operatorname{AUC}(\mu \mathrm{g} \mathrm{h} / \mathrm{ml})$ \\
\hline Fast & 0.55 & 2.91 & 8.50 \\
Fed & 2.52 & 1.21 & 19.3
\end{tabular}

acids are essential for micelle formation, they are needed for the intestinal absorption of poorly watersoluble drugs. With regard to the effect of food intake on the plasma concentration of CoQ10 after oral administration, it is possible that some food components or bile acids play important roles in the intestinal absorption of CoQ10.

In summary, we have demonstrated that a higher plasma concentration of CoQ10 was achieved by using an emulsion formulation and food intake. Beneficial effects of CoQ10 supplementation have been observed in both experimental models and human patients. ${ }^{17,18)}$ It is possible that administration of CoQ10 in an emulsion formulation enhances the pharmacological effects of CoQ10. Further studies are needed to assess the pharmacological effects of CoQ10 using an emulsion formulation and to elucidate the mechanisms by which food components or bile acids increase the bioavailability of CoQ10. Such investigations will provide important information for improving the pharmacological effects of CoQ10.

Acknowledgements We thank Kougen Co.,
Ltd. for providing CoQ10 and supporting this study.

\section{REFERENCES}

1) Ernster L., Forsmark P., Nordenbrand K., Biofactors, 3, 241-248 (1992).

2) Forsmark P., Aberg F., Norling B., Nordenbrand K., Dallner G., Ernster L., FEBS Lett., 285, 39-43 (1991).

3) Stocker R., Bowry V. W., Frei, B., Proc. Natl. Acad. Sci. U.S.A., 88, 1646-1650 (1991) .

4) Kalen A., Appelkvist E. L., Dallner G., Lipids, 24, 579-584 (1989).

5) Lalani S. R., Vladutiu G. D., Plunkett K., Lotze T. E., Adesina A. M., Scaglia F., Arch. Neurol., 62, 317-320 (2005).

6) Overvad K., Diamant B., Holm L., Holmer G., Mortensen S. A., Stender S., Eur. J. Clin. Nutr., 53, 764-770 (1999).

7) Langsjoen P. H., Langsjoen A. M., Biofactors, 9, 273-284 (1999).

8) Greenberg S., Frishman W. H., J. Clin. Pharmacol., 30, 596-608 (1990).

9) Hodgson J. M., Watts G. F., Playford D. A., Burke V., Croft K. D., Eur. J. Clin. Nutr., 56, 1137-1142 (2002).

10) Roffe L., Schmidt K., Ernst E., J. Clin. Oncol., 22, 4418-4424 (2004).

11) Zhang Y., Aberg F., Appelkvist E. L., Dallner G., Ernster L., J. Nutr., 125, 446-453 (1995) .

12) Itagaki S., Chiba M., Shimamoto S., Sugawara M., Kobayashi M., Miyazaki K., Hirano T., Iseki K., Drug Metab. Pharmacokinet., 20, 72-78 (2005).

13) Lu W. L., Zhang Q., Lee H. S., Zhou T. Y., Sun H. D., Zhang D.W., Zheng L., Lee M., Wong S. M., Biol. Pharm. Bull., 26, 52-55 (2003).

14) Bonakdar R. A., Guarneri E., Am. Fam. Physician, 72, 1065-1070 (2005).

15) Hauss D. J., Fogal S. E., Ficorilli J. V., Price C. A., Roy T., Jayaraj A. A., Keirns J. J., J. Pharm. Sci., 87, 164-169 (1998).

16) Erkko P., Granlund H., Nuutinen M., Reitamo S., Br. J. Dermatol., 136, 82-88 (1997).

17) Matthews R. T., Yang L., Browne S., Baik M., Beal M. F., Proc. Natl. Acad. Sci. U.S.A., 95, 8892-8897 (1998).

18) Beal M. F., J. Bioenerg. Biomembr., 36, 381386 (2004). 